\title{
Variation lexicale, catégorisation et convergence culturelle
}

Formes et valeurs des composés à -eleo- et -lado- « huile » en grec moderne

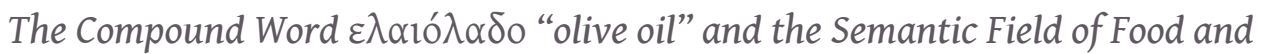
Food Habits, Explored Through such a Key Element of the Mediterranean Diet as Olive Oil

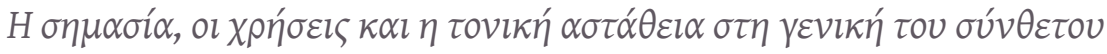

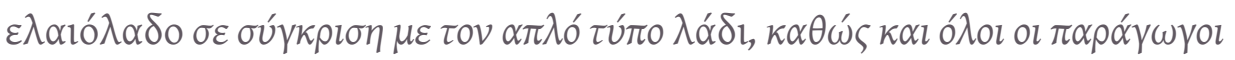

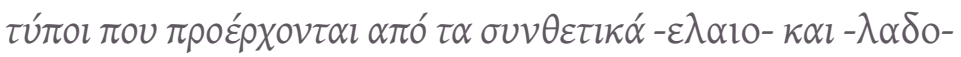

\section{Sophie Vassilaki}

\section{OpenEdition}

Journals

Édition électronique

URL : https://journals.openedition.org/ceb/7222

DOI : $10.4000 /$ ceb.7222

ISSN : 2261-4184

Éditeur

INALCO

Édition imprimée

ISBN : 978-2-85837-230-6

ISSN : 0290-7402

Référence électronique

Sophie Vassilaki, «Variation lexicale, catégorisation et convergence culturelle », Cahiers balkaniques [En ligne], Hors-série | 2016, mis en ligne le 23 mars 2017, consulté le 21 septembre 2021. URL : http:// journals.openedition.org/ceb/7222; DOI : https://doi.org/10.4000/ceb.7222

Ce document a été généré automatiquement le 21 septembre 2021.

Cahiers balkaniques est mis à disposition selon les termes de la Licence Creative Commons Attribution - Pas d'Utilisation Commerciale 4.0 International. 


\title{
Variation lexicale, catégorisation et convergence culturelle
}

\author{
Formes et valeurs des composés à -eleo- et -lado- « huile » en grec \\ moderne

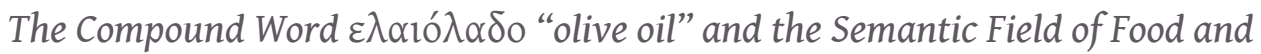 \\ Food Habits, Explored Through such a Key Element of the Mediterranean Diet as \\ Olive Oil

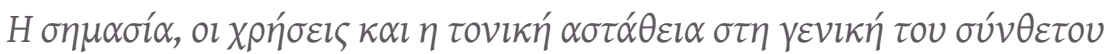

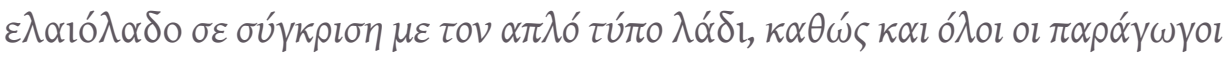

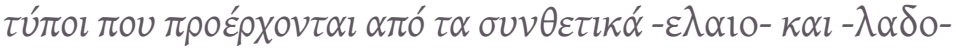

\section{Sophie Vassilaki}

\section{Introduction}

1 Cette étude s'intéresse aux formes simples et composées signifiant " huile » en grec moderne et aux valeurs sémantiques et référentielles qui leur sont associées. Nous aborderons cette thématique sous deux aspects dont le lien est établi par le paramètre de la variation:

- le mode de catégorisation lexicale des mots composés contenant le segment -huile- sous ses deux variantes - $\varepsilon \lambda \alpha 10^{-},-\lambda \alpha \delta 0^{-}$;

- le mode d'alternance du mot $\lambda \alpha \alpha \delta$ « huile»/« huile d'olive» (par défaut) et du composé $\varepsilon \lambda \alpha \iota o ́ \lambda \alpha \delta o$ « huile d'olive »; la variation accentuelle de ce dernier, notamment au génitif ${ }^{1}$, en dépit de sa structure de composé entraînant en principe la fixité de l'accent.

2 À travers la grille d'analyse proposée ici, nous allons tenter de dégager quelques pistes de réflexion sur les questions traitées allant si possible au-delà du cadre explicatif

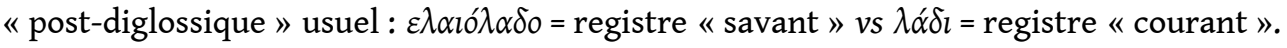
Ce critère binaire - même sur un mode gradué, il s'agit toujours d'assigner une marque de registre - et foncièrement subjectif semble en effet inadéquat comme principe de 
régulation des formes traitées ici. Nous devons par conséquent élargir la perspective et poser cette thématique dans un cadre qui tienne compte d'autres paramètres de variation. Nous allons ainsi nous intéresser à la structure et à la productivité des composés étudiés, au mode de construction de leurs valeurs référentielles et aux types de discours dans lesquels ils apparaissent. C'est dans ce cadre que la question connexe de l'alternance entre forme simple et forme composée peut être traitée. La visée de cette recherche préliminaire est de proposer un classement des composés sur la base duquel la relation entre catégorisation lexicale et représentation sémantique de ces unités dans le processus de standardisation du grec moderne pourrait être étudiée.

3 Avant de passer à l'aspect proprement linguistique de la question, il nous paraît nécessaire de présenter quelques considérations sur certaines données extralinguistiques dont le rôle semble déterminant à la prolifération des discours contenant ces formes; la bibliographie sur la question en témoigne ${ }^{2}$. Dans un contexte de globalisation et de convergence culturelle, l'exemple de l'huile, et plus généralement le champ de la nourriture et plus spécifiquement de la gastronomie, mettent en évidence l'incidence du régime de commercialisation des biens sur l'évolution des pratiques discursives, orales et écrites. Celles-ci font l'objet d'analyses au sein des études sur la diversité culturelle, sur les identités (nostalgie des racines, traditions culinaires, nouvelles attaches à la terre des classes moyennes), locale (produits du terroir), ethnique ("hellenic goods"), rurale, et cetera ${ }^{3}$. Il s'agit de textes descriptifs, narratifs, mais aussi de textes fabriqués pour la promotion des produits, des marques, des appellations contrôlées jouant sur un imaginaire collectif riche et sensible et conditionnant le changement de goût d'une partie des consommateurs. Ces textes et discours sont quotidiennement véhiculés à travers les émissions culinaires, les recettes des (grands) chef(s) pour "épater les convives» (nouvelles recettes "venues d'ailleurs" qui mettent en évidence un glissement insensible vers de nouvelles variétés), les sites web des producteurs et des industriels, la multiplication des blogs à thématique " hygiène, beauté et bien-être », etc.

4 Pour ce qui est du champ qui nous intéresse ici, un savoir-faire artisanal ancré dans les cultures locales évolue à l'ère de l'industrie huilière vers des modes de production et de commercialisation qui induisent une standardisation non seulement dans le faire, mais aussi dans le dire. Sur le plan linguistique, cela crée un cadre de circulation des discours, notamment médiatique et publicitaire, devenus des genres routiniers. À titre

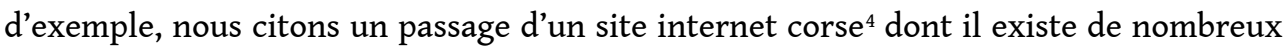
équivalents sur le web (pour la Grèce ${ }^{5}$, le Maroc, la Tunisie, etc.). Le répertoire linguistique fait appel, comme nous venons de le mentionner, à quelques stéréotypes (Antiquité, héritage, pratiques multiséculaires, civilisation, identité, art de vivre, douceur, arômes, etc.) liés à des représentations partagées en Méditerranée créant un espace référentiel («les privilèges du bassin méditerranéen ») à l'intérieur duquel les mots utilisés deviennent signifiants :

Les pratiques culturales et les variétés implantées depuis l'Antiquité sont l'héritage d'une civilisation ancestrale basée sur la culture de l'olivier; comme le vin, l'huile d'olive est l'un des plus anciens produits alimentaires [...].

L'huile d'olive de [...] est un produit identitaire par excellence, issue en grande partie d'arbres multicentenaires. De tout temps en [...] l'on a cultivé l'olivier et produit une huile d'olive à partir de fruits mûrs. Aujourd'hui, la récolte traditionnelle sur filets par chute naturelle des arbres multiséculaires et la récolte mécanique sur de jeunes vergers de variétés locales se côtoient et se complètent 
pour offrir une huile d'olive de [...] en AOP [...] caractérisée par sa douceur, pour deux tonalités d'arômes [...].

5 C'est dans ce cadre que le mécanisme de la variation qui nous intéresse ici peut être étudié. C'est notamment dans ces discours que l'on observe le croisement des «termes» - en mode répertoire et à valeur prédéfinie - et des " mots de la langue » dont la valeur référentielle n'est pas fixée a priori, mais reconstruite et stabilisée par le contexte discursif.

\section{Trois types de composés}

6 Après ces considérations générales, venons-en aux données sur la combinatoire des deux bases - $\varepsilon \lambda \alpha{ }_{10}$ - et $-\lambda \alpha \delta o$ - issues principalement des dictionnaires ${ }^{6}$. L'ensemble de ces composés relève de la classe des "mots construits " selon la terminologie établie par D. Corbin (1987), dans la mesure où leur sens est prédictible car entièrement compositionnel par rapport à leur structure interne. Pour ce qui est de la typologie de leur structure interne, i.e. de la relation sémantico-syntaxique constitutive des unités NN, les composés étudiés ici relèvent majoritairement mais non exclusivement de la classe des subordinatifs ${ }^{7}$, dans la mesure où la relation NN est de type repérérepère, à ordre contraint. Nous nous limiterons ici à un examen des formes considérées sans entrer dans les détails de la discussion sur la composition néo-hellénique qui a fait l'objet de nombreuses analyses (cf. bibliographie indicative) ${ }^{8}$.

7 Les composés qui nous intéressent ici se répartissent en trois catégories distinctes :

\section{1. Composition à base de la forme - $\varepsilon \lambda$ aı - dite savante, identifiable sur le plan phonétique par la séquence VCVV sans synizèse}

8 Cette forme peut apparaître aussi bien comme premier composé que comme second :

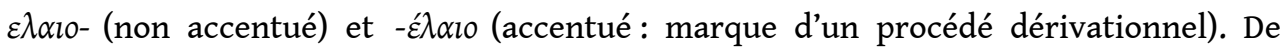
nombreuses bases compositionnelles présentent le même trait, les mots liés à la nourriture et indirectement aux procédés de préparation ou de mélange des ingrédients sont particulièrement représentés, par ex.: $\rho v \zeta o ́-\gamma \alpha \lambda o, \rho v \zeta-\alpha \lambda^{\prime} \varepsilon v \rho o$, $\sigma \pi \alpha v \alpha \kappa o_{-} \rho v \zeta o, \pi \rho \alpha \sigma o^{-} \rho \nu \zeta o$, mais aussi $-\psi \alpha \rho 0^{-},-\delta \varepsilon v \tau \rho 0^{-},-\varphi v \lambda \lambda_{0-}{ }^{9}$, etc.

EAAIO- (« olive/olivier », « huile », « huile d'olive »)

9 Premier composé, environ 40 composés, classe productive. Indépendamment des particularités de la structure interne de chaque unité NN, ce premier composé opère ici sur un mode de détermination existentiel: il y a de l'huile/olive(s). En ce sens, il désigne un exemplaire d'une propriété qui devient ainsi une catégorie générale. La valeur obtenue par ce mode de détermination est donc celle d'une désignation; il y a renvoi à la notion abstraite où olive/olivier/huile sont indistincts dont $\varepsilon \lambda \alpha 10$ - est un exemplaire (une simple « occurrence de »), permettant la mise en place d'un répertoire ouvert, comme attesté par la productivité de cette forme.

Dans son Recueil de mots nouveaux forgés par les lettrés, etc., paru en 1900, Stéphanos Koumanoudis ${ }^{10}$ recense (entre 1860 et 1895 ) au total 98 composés avec $\varepsilon \lambda \alpha i 0-$ comme 


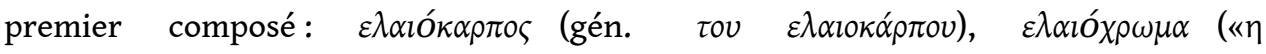

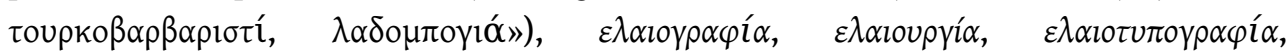

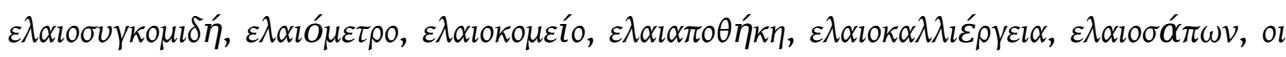
$\varepsilon \lambda \alpha$ lok $\lambda \dot{\varepsilon} \pi \tau \alpha$ l, etc., dont un bon tiers est hors d'usage aujourd'hui.

Ce sont des composés subordinatifs, non attributifs ( $\mathrm{x}$ est $\mathrm{y}$ ); notons que dans ce type de composition savante, il n'y a pas de composés coordinatifs (? ils désignent des

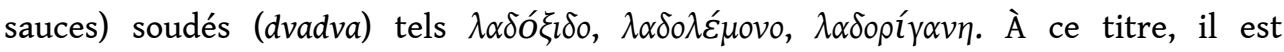
intéressant de relever le recensement par Koumanoudis du mot

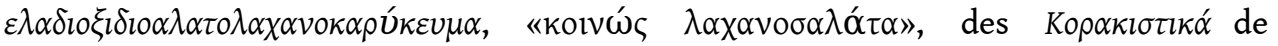
Iakovakis Rizos Neroulos, paru en 1812. Pour ce composé, forgé sur des modèles anciens (Aristophane), et plus récents, français, bien connus, c'est bien le composé « médiéval » non-savant $\varepsilon \lambda \alpha \delta$ o- qui apparait.

Pour ce qui est de la structure interne de ces composés, ils se répartissent dans deux classes:

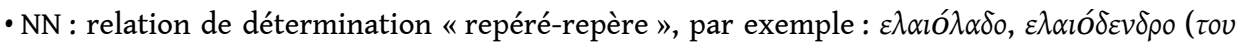

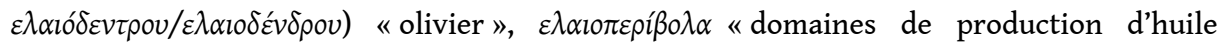

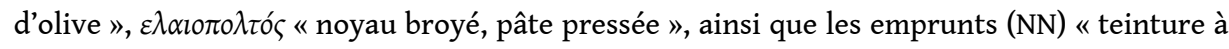
l'huile».

- NN processif (déverbal/dérivationnel) : relation de type prédicatif (verbe-argument):

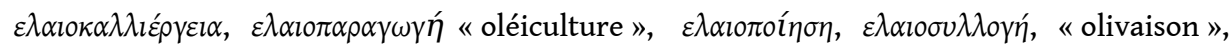

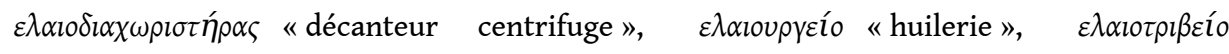

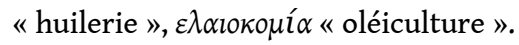

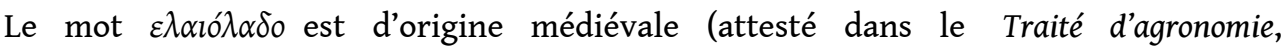
d'Agapios Landos, 1643) ${ }^{11}$; d'après la première édition du dictionnaire de Babiniotis, il n'est pas pluralisable, alors que le dictionnaire de la Fondation Triandaphyllidis indique le contraire. L'emploi du mot au pluriel est en effet bien attesté, mais il est récent; il renvoie aux types d'huile, à l'huile frelatée (plusieurs occurrences) ainsi qu'aux récipients, par exemple :

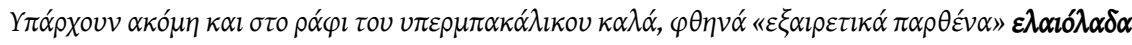

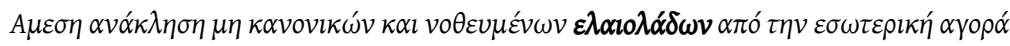

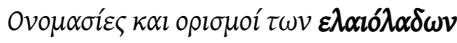

L'attestation régulière du mot $\varepsilon \lambda \alpha i o ́ \lambda \alpha \delta o$ depuis les années 1930 est concomitante au conditionnement de l'huile d'olive en bidons métalliques et à cette nouvelle forme de commercialisation et de consommation que cela implique. Il s'agit donc non pas d'un mot nouveau, mais d'un nouvel objet: les bidons métalliques joliment décorés (en couleur), devenus objets du quotidien, mais aussi objets cultes. Le mot dans cette nouvelle acception s'oppose à l'huile en vrac $(\chi u ́ \mu \alpha)$, de qualité et de pureté douteuses (frelatage). À ce titre, il est intéressant de noter que $\varepsilon \lambda \alpha \imath$ '́ $\alpha \delta o$ dans ces premières attestations est systématiquement précédé de qualificatifs attestant de son authenticité

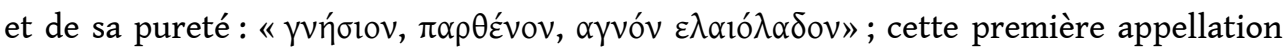

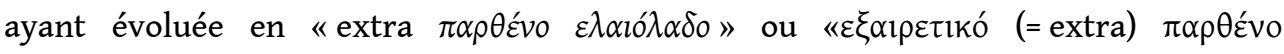

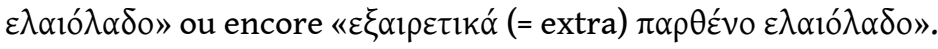

15 Ajoutons à cette liste des composés les néologismes de type calque attestés dans la

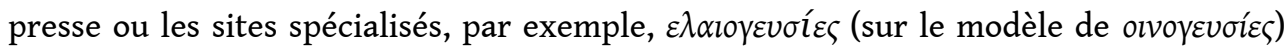

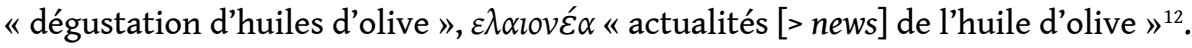




\section{$-E \Lambda A I O(-\varepsilon ́ \lambda a ı O)$} 80 composés). La productivité récente de cette forme est sans doute due à des facteurs extralinguistiques. Pour ce qui est des huiles consommables, si l'on s'éloigne de la Méditerranée, l'huile d'olive ne se place qu'au $6^{\mathrm{e}}$ rang des productions des principales huiles végétales après l'huile de soja, de tournesol, de colza, d'arachide et de coton ${ }^{13}$. Par ailleurs, le changement de goût des consommateurs, y compris pour les huiles non comestibles, mais utilisables en cosmétique, est annonciateur d'un avenir vers de nouveaux types de produits et vers des variétés à base de graines oléagineuses de l'industrie huilière. partir de la notion générique /- $\varepsilon \lambda \alpha 10-/$, mais référentiellement centrée sur l'huile d'olive créant dans ce type de composition une sous-classe: huiles autres que (alternatives) « huile d'olive ».

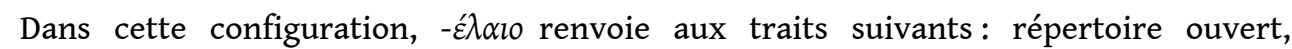
étiquetage, identification du produit, origine et composition, \pm prestige/qualité, information consommateur, danger/prévention ${ }^{14}$. Pour ce qui est de la structure

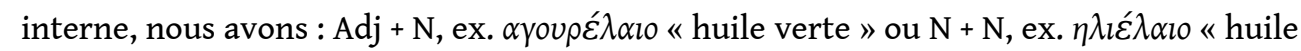

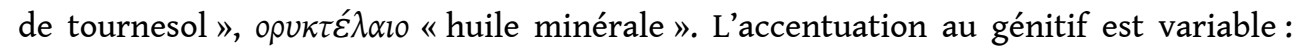

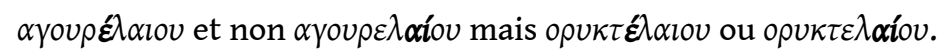

19 Selon les modes de consommation et la familiarité avec la variété, ces composés

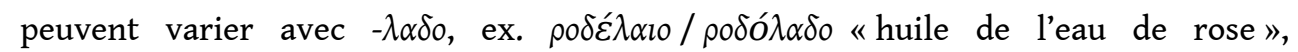

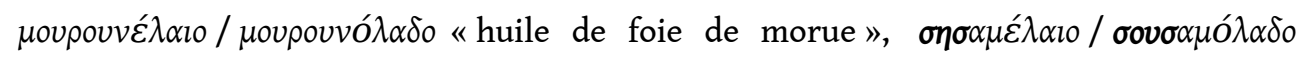
" huile de sésame », $\beta \alpha \mu \beta \alpha \kappa \varepsilon ́ \lambda \alpha$ lo / $\beta \alpha \mu \beta \alpha \kappa o ́ \lambda \alpha \delta o$ et $\mu \pi \alpha \mu \pi \alpha \kappa o ́ \lambda \alpha \delta o$ " huile de coton », cf. par ailleurs $\imath \chi \theta v \varepsilon ́ \lambda \alpha$ lo mais $\psi \alpha \rho o ́ \lambda \alpha \delta o$ « huile de poisson ». Notons enfin les lexicalisés

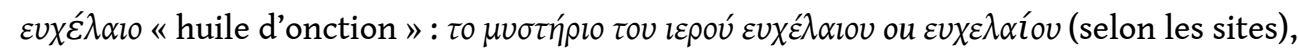

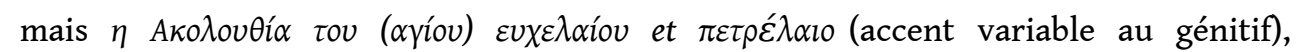
traduction du français pétrole < petroleum.

\section{2. Composition « non savante »}

\section{$\wedge A \Delta O-$}

Premier composé, environ 15 composés de type $\mathrm{N}+\mathrm{N}$. Ce composé ne renvoie pas à l'existence d'huile (il y a de...) comme dans le cas I., mais il situe l'unité NN dans la classe d'objets dont "l'huile», en tant qu'entité-type, est le centre organisateur. «Huile» ici est quasiment toujours assimilée à l'huile d'olive. Cette classe ne désigne pas des variétés d'huiles végétales ou autres, mais des entités singulières parmi d'autres, contenues dans cette même classe; à ce titre, elles peuvent faire l'objet d'une évaluation ou d'une comparaison. Cette référence à un type structure tout un espace de représentations lié à l'huile, mais aussi à tout ce qui, parmi les matières grasses, est autre que « huile» (beurre, etc.) : observer le jeûne, manger sain et léger (pas de gras animal), par exemple : $\lambda \alpha \delta$ okoúlovpo " gâteau sec à l'huile ", $\lambda \alpha \delta o ́ \psi \omega \mu \nu$ " pain à l'huile »

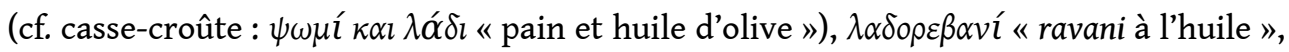
$\lambda \alpha \delta o \tau \hat{v} \rho \imath$ "fromage conservé à l'huile d'olive " ${ }^{15}$, préparations simples/basiques mais

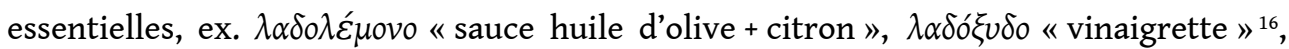


$\lambda \alpha \delta o \rho i ́ \gamma \alpha v \eta$ "(sauce) huile et origan ", protection/résistance, récipients/objets dédiés à statut particulier, ex. $\lambda \alpha \delta$ $\alpha \pi$ roүı́ « peinture à l'huile », $\lambda \alpha \delta o ́ \kappa o \lambda \lambda \alpha / \lambda \alpha \delta o ́ \chi \alpha \rho \tau \tau$ « papier de cuisson/à l'huile", $\lambda \alpha \delta o ́ \pi \alpha v o$ "drap-serviette de baptême", $\lambda \alpha \delta o u \pi o u ́ \kappa \alpha \lambda_{0}$

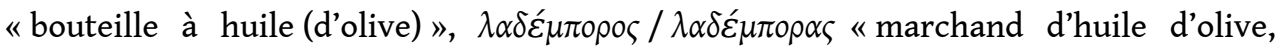
grossiste (mais $\chi \alpha \rho \tau \varepsilon \dot{\varepsilon} \mu \pi \circ \rho \circ \varsigma /{ }^{*} \alpha \varsigma$ ).

Cette valeur qualitative attribuée aux différents supports les distingue ainsi des autres, ceux qui ne sont pas à l'huile (par défaut, d'olive) qui sont quasi éliminés de l'espace de représentation. Le segment $\lambda \alpha \delta o$ - prend en quelque sorte le pouvoir sur le $\mathrm{N}$ et sort de sa fonction première de déterminant "huile» y compris dans des cas comme $\lambda \alpha \delta$ ouroyıá (à base d'huile de lin, de carthame ou de noix) qui évoque l'ultime couche huileuse en guise de protection, i.e. une représentation propre au domaine de l'huile d'olive (pouvoir figuratif des lexèmes).

\section{$\Lambda A \Delta O$}

Deuxième composé, env. 40 composés (classe plus productive en postposition, analogue

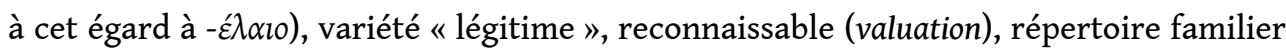
« interne » à la représentation de l'huile. Il n'y a pas d'effet d'étiquetage comme dans la classe à - $\varepsilon \lambda \alpha l o$; ces variétés sont perçues plutôt comme des avatars, i.e. des incarnations possibles d'huile (ayant toujours comme type l'huile d'olive), autrement dit, l'identité de chaque type d'huile n'est pas reformulée comme nouveau rapport à la catégorie (cf. - $\dot{\lambda} \alpha$ lo) dont elle constituerait un exemplaire.

C'est précisément dans cette classe que l'on trouve le plus de variation dans le premier composé, par exemple $\beta \alpha \mu \beta \alpha \kappa o ́ \lambda \alpha \delta o$ / $\mu \pi \alpha \mu \pi \alpha \kappa o ́ \lambda \alpha \delta \delta$, mais seulement $\beta \alpha \mu \beta \alpha \kappa \varepsilon ́ \lambda \alpha$ lo,

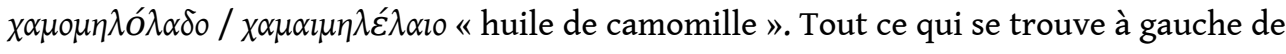
$\lambda \alpha \delta$ o- n'est donc pas contraint pour le registre, d'où $\varepsilon \lambda \alpha$ có $\lambda \alpha \delta o$ lui-même et $\lambda$ tól $\alpha \delta \delta$.

\section{3. Composition (1 syll.) « non savante », de registre littéraire, populaire, marketing}

$110-$

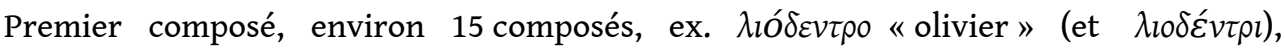

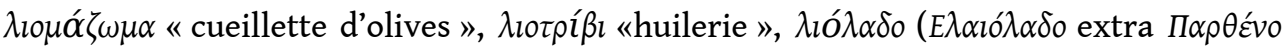

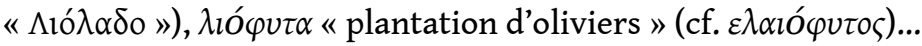

$\neq \Lambda I O-$

Premier composé $(\eta) \lambda_{\imath o-}$ "soleil» (homonyme), unités lexicalisées, ex.: $\lambda_{\imath o \pi v ́} \rho t$

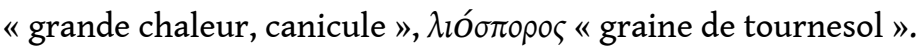

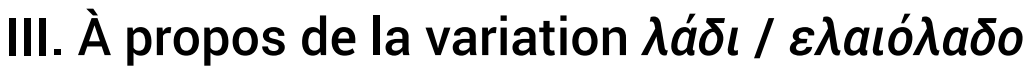

À travers l'examen des quatre constituants des unités NN étudiées, nous avons déjà dégagé quelques pistes d'interprétation de la différence sémantique entre le mot simple

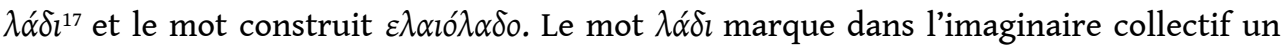
produit typique et singulier, qui partage de façon égale sa place avec le pain et le vin 
dans la «trinité fondamentale » de l'alimentation des peuples méditerranéens ${ }^{18}$. L'huile d'olive a été seule, pendant de nombreuses années, à faire l'objet du commerce des huiles en Grèce comme dans les grands centres de commerce en Méditerranée. La singularité du produit, qui est une huile de fruit (rappelons que arbre et fruit sont marqués par un seul lexème en grec, $\varepsilon \lambda_{l} \alpha$ ) et non de graines et qui est pratiquement la seule huile qui peut être consommée à l'état naturel, a pu déterminer la sémantique de $\lambda \alpha ́ \delta l$ dans son emploi par défaut pour désigner l'huile d'olive.

Rappelons ici l'existence des nombreux lexèmes et locutions construits à partir de ce radical qui mettent en évidence la richesse et la complexité du champ sémantique : vie $^{19} /$ énergie, protection/pansement, surface plane, lisse, sans aspérité, sans obstacle ou au contraire, tache, piège, mauvais genre, corruption, difficulté/épuisement ( $\mu v v$

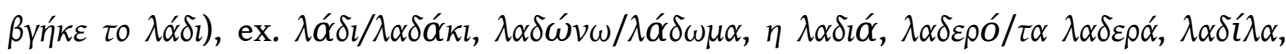

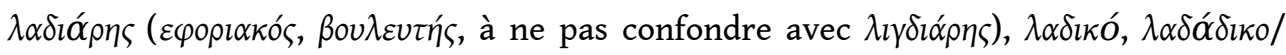
$\lambda \alpha \delta \alpha ́ \delta i \kappa \alpha$, etc.

Le mot simple est par ailleurs largement utilisé dans des textes à visée commerciale, dans les discours médiatiques ou des textes de présentation standardisée en cooccurrence (reprise, spécification) ou en alternance avec le mot construit (référence homérique, sans citation, obligatoire). Dans ce cas, le produit est marqué dans sa singularité d'«or liquide» est marqué dans sa singularité (propriété incarnée et individuée) et corrélativement opposé à tout ce qui est extérieur à cette propriété :

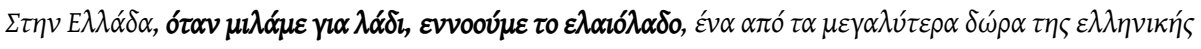

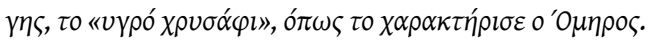

<http://www.vita.gr/diatrofi/eating-healthy/article/13473/11-erwthseis-gia-to-elaiolado/>,

[consulté le 17 décembre 2015]

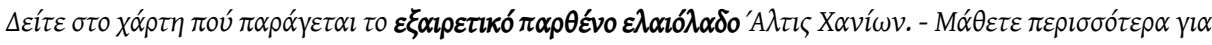

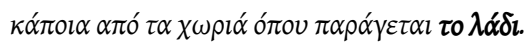

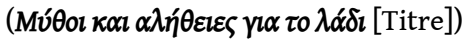

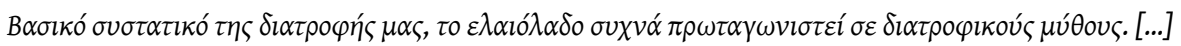

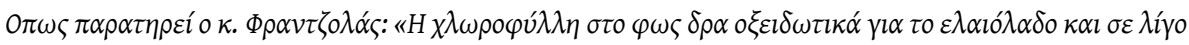

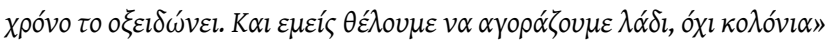

<http://www.tovima.gr/science/article/?aid=518862>, [consulté le 17 décembre 2015]

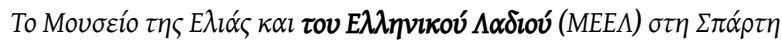

<http://www.oliveoilmuseums.gr/ecportal.asp?id=55\&nt=18\&lang=1>, [consulté le

17 décembre 2015]

29 La position du syntagme en complément nominal dans le dernier exemple, facilite ici l'emploi du mot simple ; cela permet en effet d'éviter, dans le titre, le génitif $\varepsilon \lambda \alpha$ co $\lambda \alpha ́ \delta o v$ dont l'accentuation varie. En revanche, dans le texte de présentation, $\lambda \alpha \delta^{\prime} \delta_{l}$ et $\varepsilon \lambda \alpha \iota{ }^{\prime} \lambda \alpha \delta o$ alternent, selon les principes du mécanisme décrit plus haut ${ }^{20}$.

De tout ce qui vient d'être dit, le fonctionnement et la valeur du mot construit $\varepsilon \lambda \alpha \iota$ íł $\alpha \delta$ peuvent être aisément circonscrits. Son emploi actuel très proliférant est directement lié à la grande circulation des textes dans lesquels il est fait mention d'huile d'olive en tant que terme, i.e. sans variation interne de sens, un calque de "olive oil", et, comme nous l'avons dit dès le début de cette étude, en tant que produit conditionné, de qualité contrôlée, susceptible de générer du "Label wording". Il s'agit bien d'un "exemplaire contrôlé» d'huile d'olive; son identité est (re-)vérifiée par 
rapport à la propriété dont il est la manifestation, il devient dans ces conditions un produit concurrentiel :

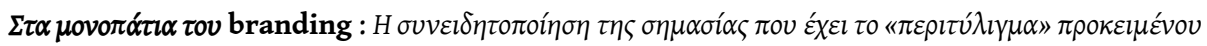

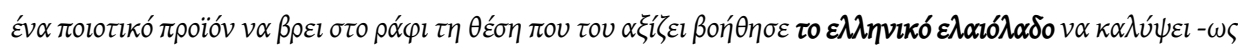

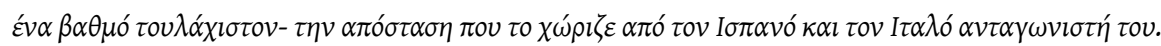
<http://www.agronews.gr/files/1/PDF/EL/11_13_EL.pdf>, [consulté le 17 décembre 2015]

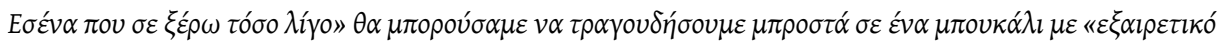

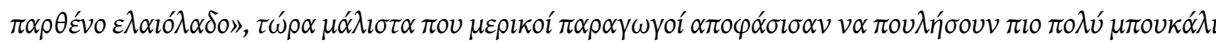

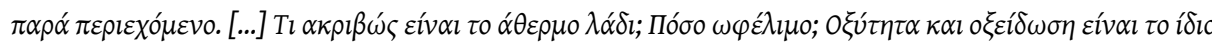
$\pi \rho \alpha ́ \gamma \mu \alpha ;$ По

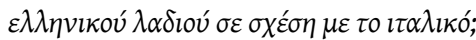
<http://www.tovima.gr/science/article/?aid=518862>, [consulté le 17 décembre 2015]

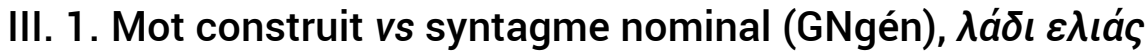

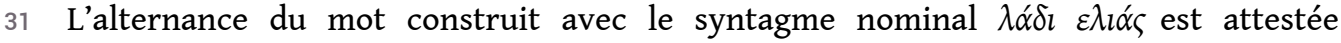
principalement dans les textes journalistiques (magazines) ou blogs à thématique cosmétique ou culinaire. La configuration récurrente est celle en mode brève «dissertation » sur un sujet en principe connu (relation entre huile d'olive et santé/

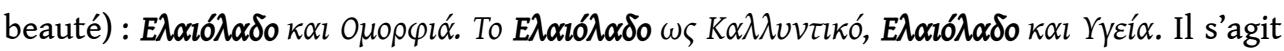
principalement d'adaptations de textes originaux en anglais ou en français.

Le syntagme nominal apparait, lui, dans le titre, lorsqu'il s'agit de donner des tips en soins et beauté ou en cuisine ( $2^{\mathrm{e}}$ pers. du singulier) indiquant des propriétés présentées

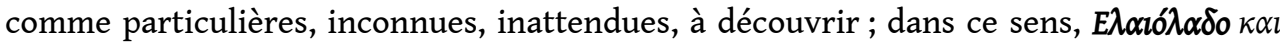

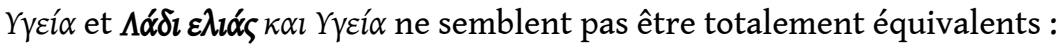

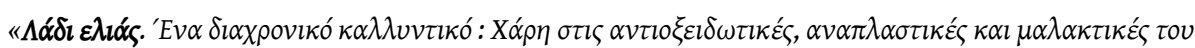

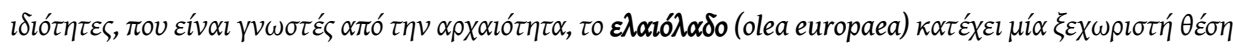

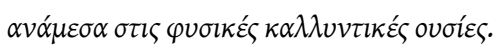

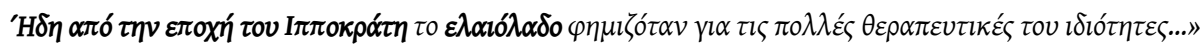
<http://www.vita.gr/beauty/doityourself/arthro/2090/ladi-elias-ena-diaxroniko-kallyntiko/>, [consulté le 17 décembre 2015]

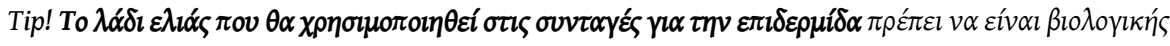

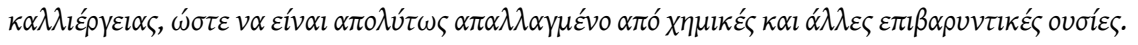
(idem)

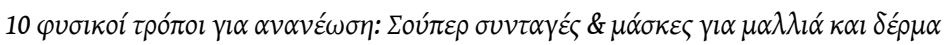

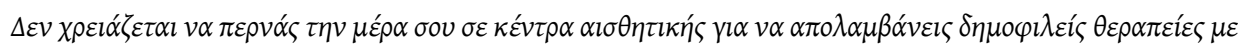

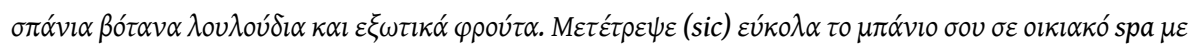

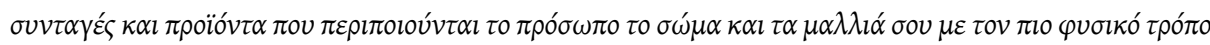

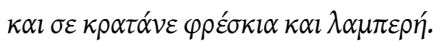

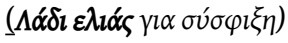

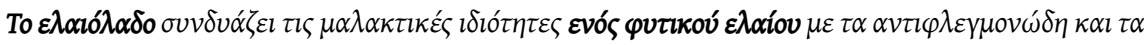

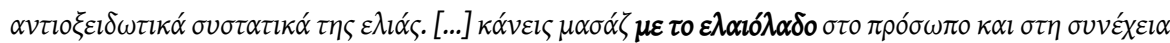

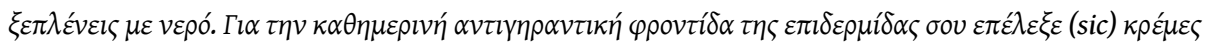

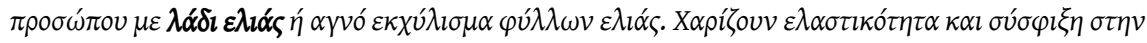

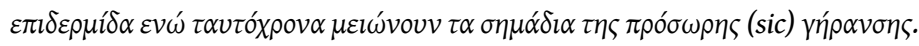

<http://www.missbloom.gr/omorfia/makigiaz-peripoiisi/10-fisiki-tropi-ya-ananeosi-sipersidages-maskes-ya-mallia-ke-derma/>, [consulté le 17 décembre 2015] 


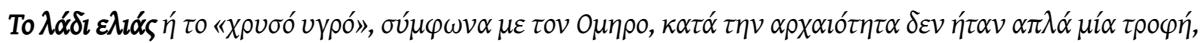

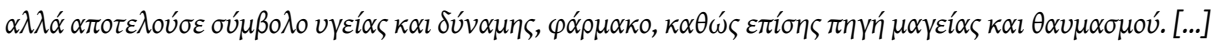

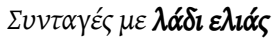

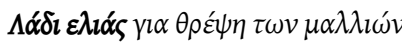

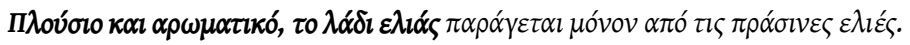

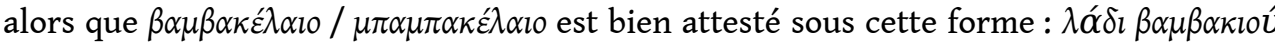

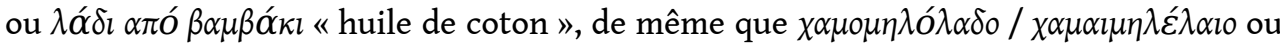

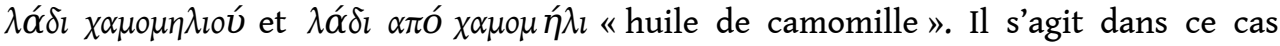
d'huiles essentielles ou d'essences végétales obtenues par des procédés différents de ceux utilisés pour l'extraction d'huile d'olive. Le groupe prépositionnel ne désigne pas dans ce cas une propriété affectée au nominal qui est à la tête du syntagme et qui le

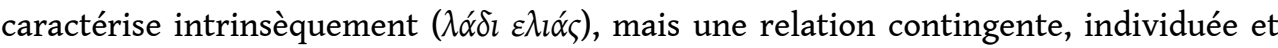
ouverte à une série de formes (Prép + acc.) susceptibles d'instancier cette position.

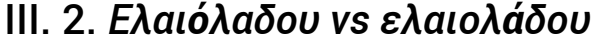

Dans cette dernière section, nous traiterons brièvement de la variation accentuelle au génitif, singulier et pluriel, du mot construit $\varepsilon \lambda \alpha i o ́ \lambda \alpha \delta o$ qui, en tant que composé, devrait fixer son accent premier, selon la règle du grec moderne : $\varepsilon \lambda \alpha i o ́ \lambda \alpha \delta o v$. Or nous savons que la forme à accentuation paroxytone $\varepsilon \lambda \alpha \imath$ ¡ó $\delta o v$ est bien attestée et qu'elle semble même plus fréquente (sondage à consolider); voici quelques exemples des deux cas, sans ou avec déterminant :

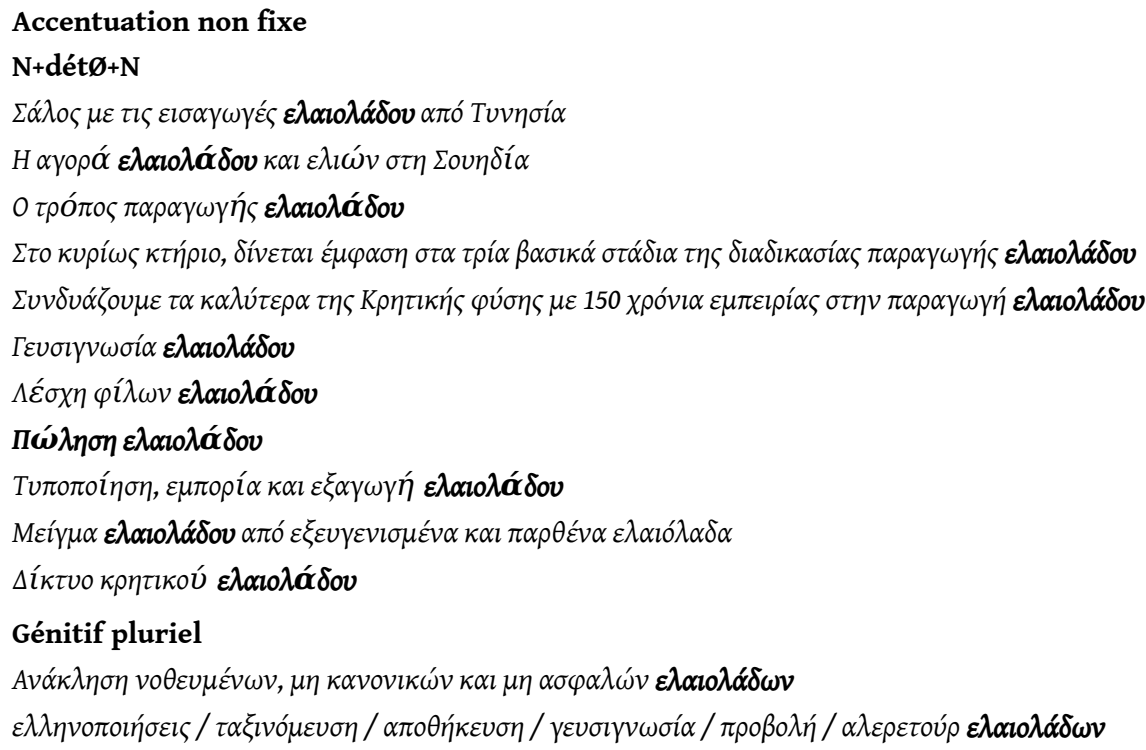




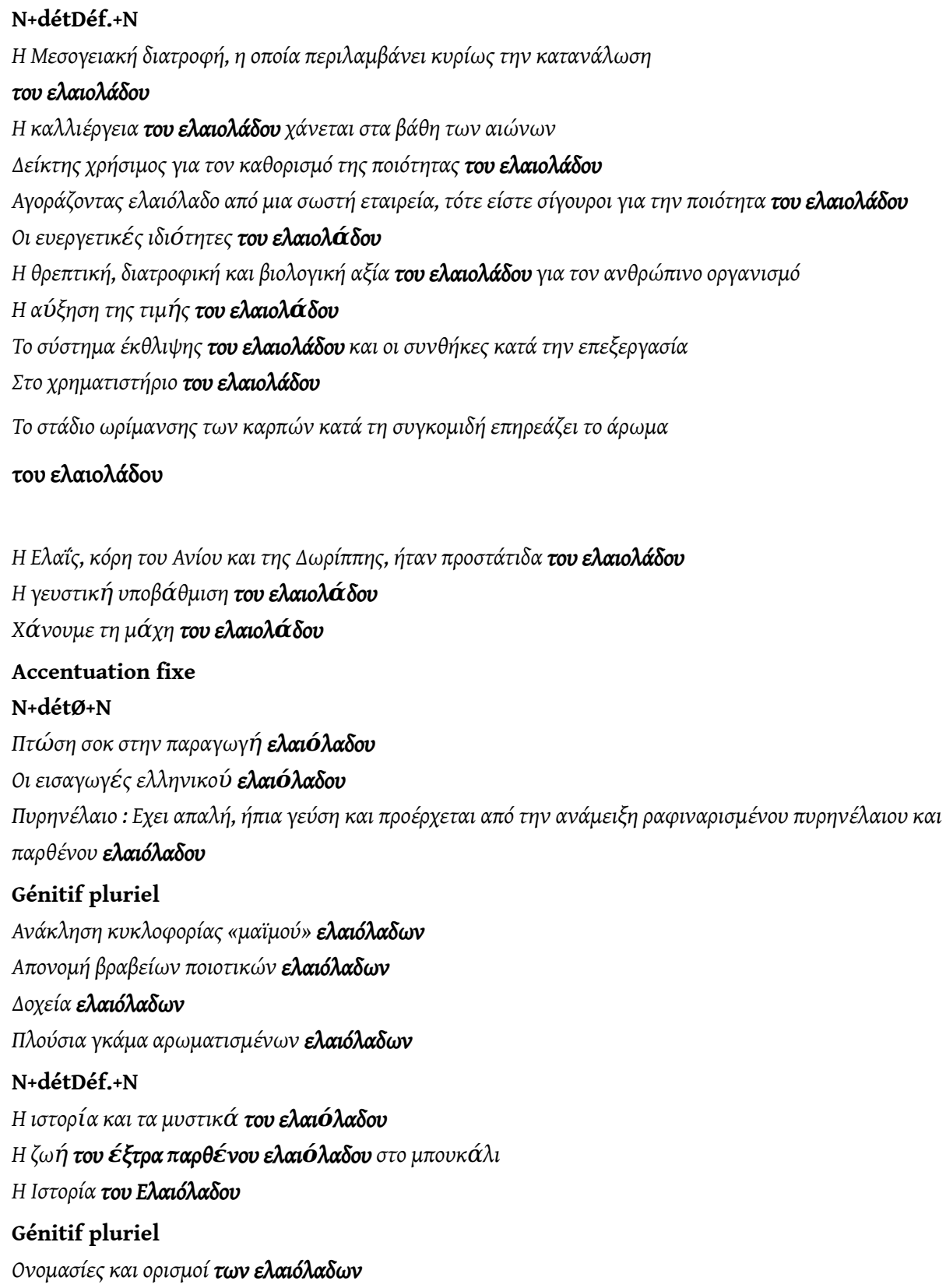

L'accentuation non fixe (descente de l'accent) traduit donc le fait que $\varepsilon \lambda \alpha$ ló $\alpha \alpha \delta o$ n'est pas perçu comme composé, mais comme unité simple. D'une autre côté, l'analyse de Giannis Haris (cf. billet cité en note 1) semble recevable : $\varepsilon \lambda \alpha$ lól $\alpha \delta o$ mot « nouveau » est

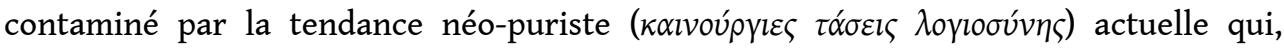
souvent sur un fond d'ignorance des règles et/ou d'insécurité linguistique (passé diglossique), est à l'origine d'une production importante de formes hypercorrigées. La contamination constitue certes un argument valable, car dans plusieurs séquences quasiment identiques ${ }^{21} \varepsilon \lambda \alpha i o ́ \lambda \alpha \delta o$ est accentué différemment, mais il ne semble pas suffisant ; l'explication du phénomène est sans doute multifactorielle. Les genres des textes, les conditions d'emploi, ainsi que la proximité interprétative de la forme $\varepsilon \lambda \alpha \imath o ́ \lambda \alpha \delta o$ avec le mot simple $\lambda \alpha ́ \delta$ peuvent aussi agir comme facteurs. En dehors de la conscience linguistique des utilisateurs (auteurs, locuteurs), on peut faire l'hypothèse que plus l'emploi du mot construit est marqué comme exemplifiant (label) plus l'accentuation du génitif aura tendance à être paroxytone (lexème non composé). 
Inversement, plus cet emploi sera marqué comme singularisant (proche du sémantisme du mot simple), plus l'accentuation du génitif sera fixe. Cette piste pourrait par ailleurs fournir une explication à l'attestation considérablement plus fréquente de la forme à accent non fixe.

\section{Conclusions}

Les composés autour des segments signifiant «huile» distinguent, créent des catégories, évoquent des images; elles constituent des déterminations autour de l'huile fondées sur des principes généraux qui participent à la structuration du lexique du grec moderne au-delà du registre des mots.

L'identité sémantique du mot construit $\varepsilon \lambda \alpha i o ́ \lambda \alpha \delta o$, qui, comme nous l'avons vu, fait partie d'un réseau de formes signifiant «huile» est construite sur au moins trois strates d'emplois :

- Le nouveau mot construit, terme technique, attesté dans un traité savant d'agriculture/ agronomie dans les années 1630 ;

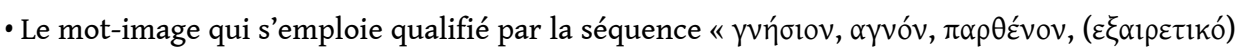
...» (authenticité et qualité), dans ce nouveau mode d'existence que représente le conditionnement dans un bidon métallique, objet si présent par ailleurs dans les maisons grecques et produit magnifiquement recyclé depuis qu'il existe.

- Un nouveau lexème, dans un emploi qui tend à effacer son identité morphologique première

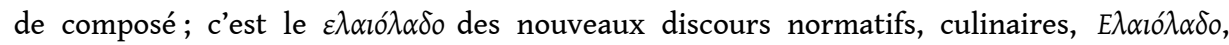

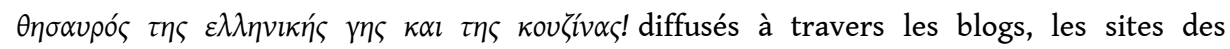
professionnels du secteur. E $\lambda \alpha$ ló $\alpha \alpha \delta o$ devient alors un mot d'une nouvelle koinè, aussi bien gastronomique que linguistique, un équivalent parfait de "olive oil", identité qui facilite sa promotion dans un espace très concurrentiel.

\section{BIBLIOGRAPHIE}

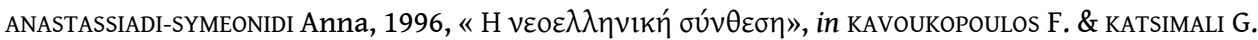

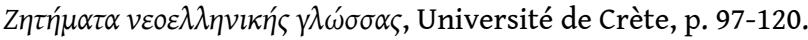

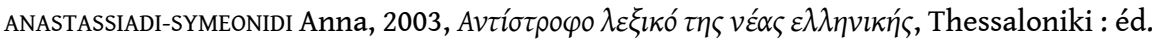

Fondation Manolis Triandaphyllidis,

<http://www.greek-language.gr/greekLang/modern_greek/tools/lexica/reverse/>.

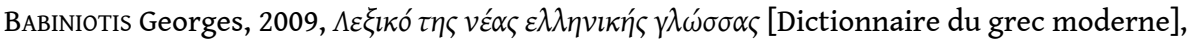

Athènes : éd. Centre de lexicologie.

ETBA [Banque nationale de développement industriel], 1996, $E \lambda_{l} \alpha \dot{\alpha} \kappa \alpha_{l} \lambda \alpha_{\alpha} \delta_{l}$, Actes du $4^{\mathrm{e}}$ atelier de

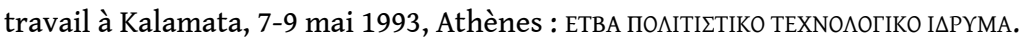

GAVRIILIDOU Zoé, 1997, Étude comparative des suites NN en français et en grec. Élaboration d'un lexique bilingue, Lille : éd. Presses Universitaires du Septentrion. 
IREMAM [Institut de recherches sur le monde arabe et musulman], 1983, L'huile d'olive en Méditerranée ; Histoire, anthropologie, économie de l'Antiquité à nos jours. Nouvelle édition en ligne, http://books.openedition.org/iremam/692, consulté le 17 décembre 2015.

LIEBER Rochelle \& ŠTEKAUER Pavol, 2011, The Oxford Handbook of Compounding, Oxford University Press.

MATTHAIOU ANNA, 1997, Aspects de l'alimentation en Grèce sous la domination ottomane : des réglementations au discours normatif, coll. Studien zur Geschichte Südosteuropas, éd. P. Lang.

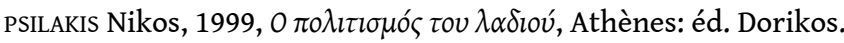

RALLI Angela, 2013, Compounding in Modern Greek, Studies in Morphology 2, Springer.

SCALISE Sergio, BISETTO Antonietta \& GUEVARA Emiliano, 2005, "Selection in compounding and derivation" in Wolfgang U. DRESSLER, Dieter KASTOVSKY, Oskar E. PFEIFFER, Franz RAINER (eds.), Morphology and Its Demarcations, Amsterdam/Philadelphia: Benjamins, pp. 133-150.

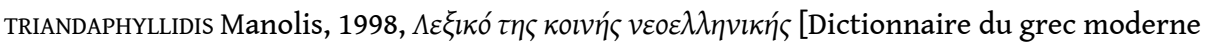

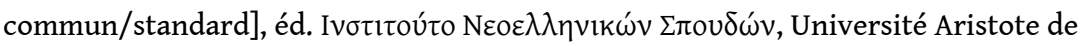
Thessalonique : I $\delta \rho u \mu \alpha$ M $\alpha$ vó $\lambda \eta$ T $\rho \alpha v \tau \alpha \varphi v \lambda \lambda i ́ \delta \eta$ [Institut d'études néo-helléniques/Fondation Manolis Triandaphyllidis].

YIAKOUMAKI Vassiliki, 2006, “'Local,' 'Ethnic,' and 'Rural' Food: On the emergence of 'Cultural Diversity' in Post-EU-Accession Greece”, in Journal of Modern Greek Studies 24, The John Hopkins University Press, pp. 415-445.

\section{NOTES}

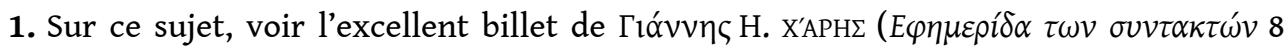

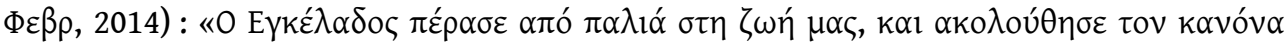

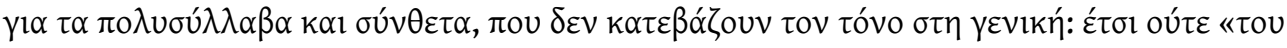

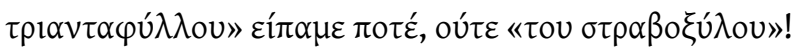

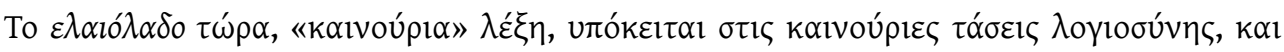

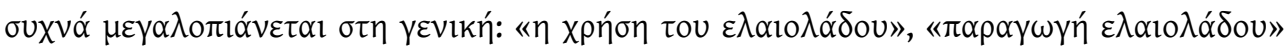

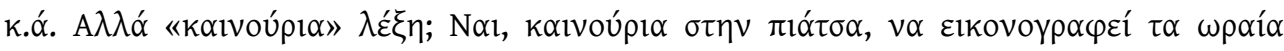

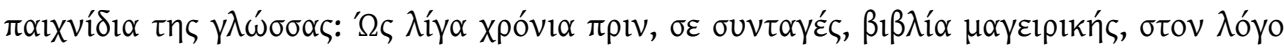

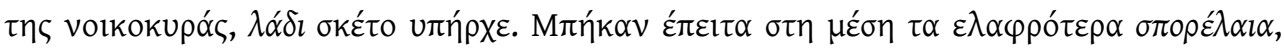

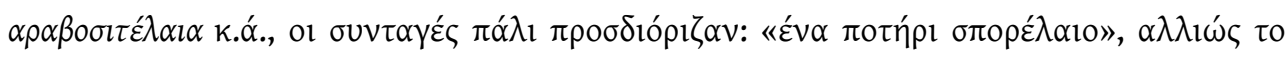

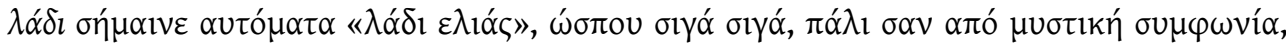

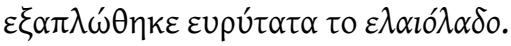

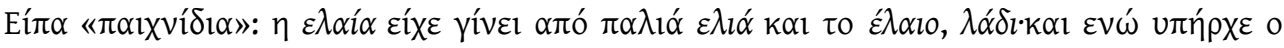

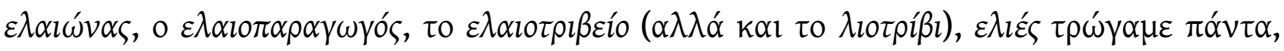

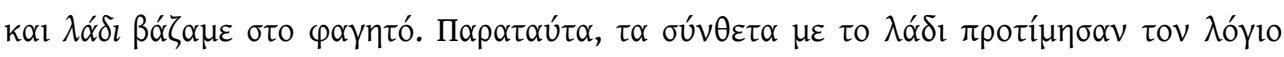

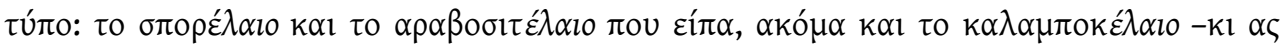

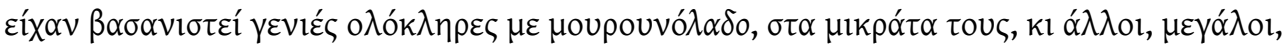

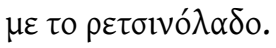

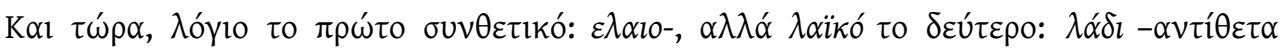

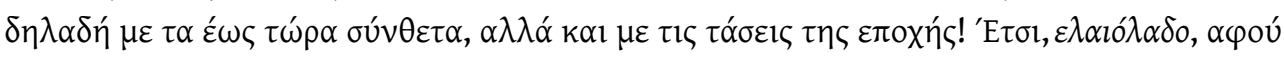

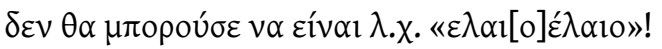




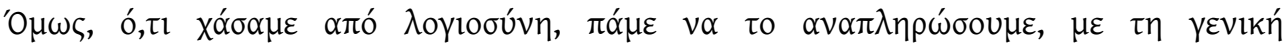

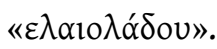

'E $\lambda \alpha$ เос!»»

2. Cf., à titre d'exemple, MatThaiou 1997 ; PSILAKIS 1999 ; éd. IREMAM 1983 ; éd. ETBA 1996.

3. Cf. sur ce point, YIAKOUMAKI 2006.

4. http://www.oliudicorsica.fr/olive-savoir_faire_ancestral-26.html, consulté le 17 décembre 2015.

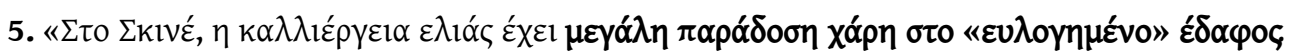

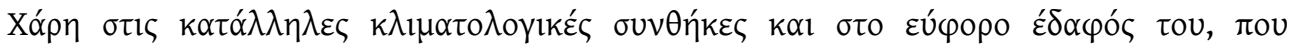

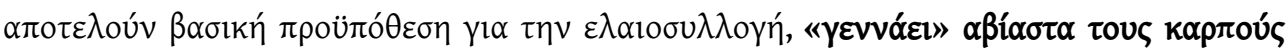

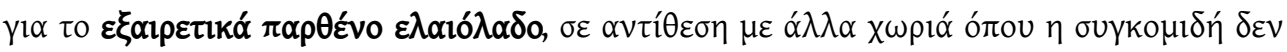

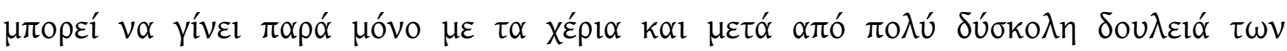

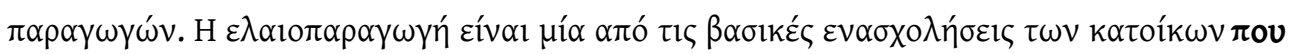

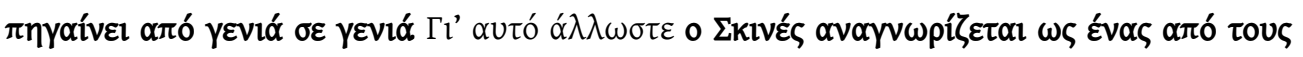

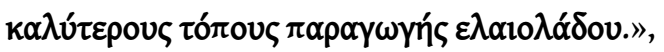

http://www.altis.com.gr/chania/skines.php, consulté le 17 décembre 2015.

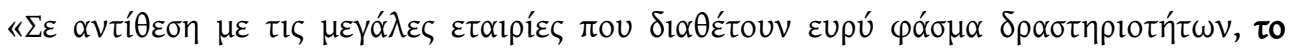

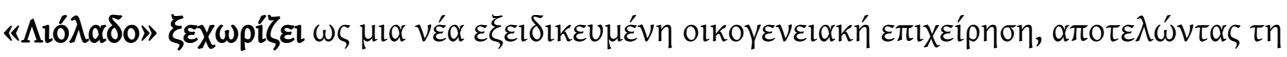

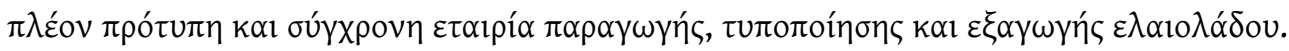

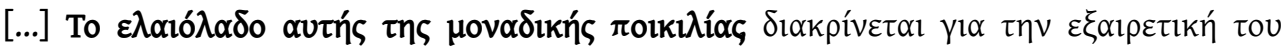

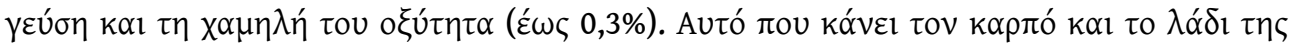

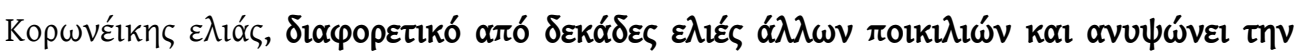

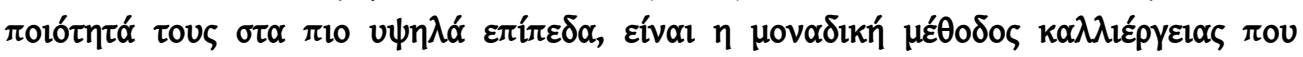

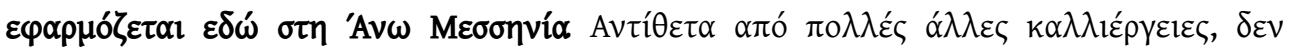

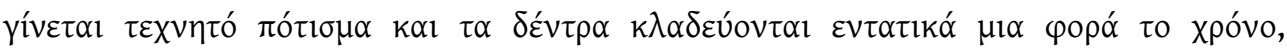

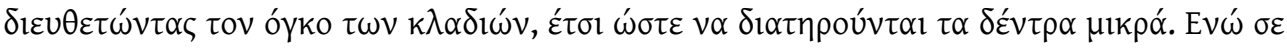

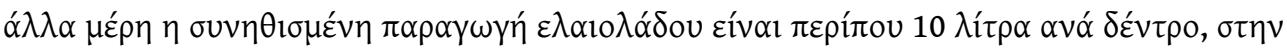

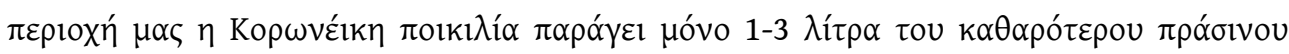

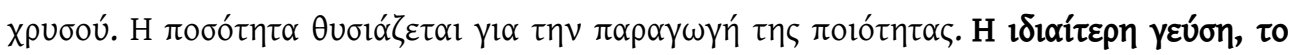

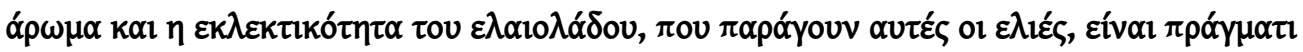

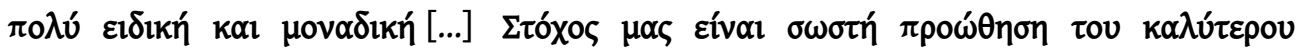

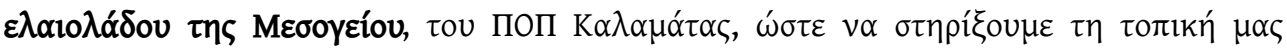

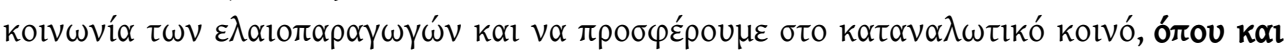

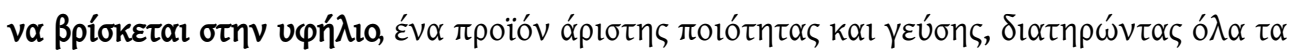

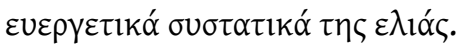

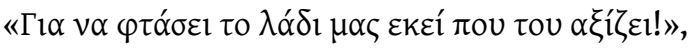
http://www.liolado.gr/index.php?lang=el, consulté le 17 décembre 2015.

6. Cf . ANASTASSIADI-SYMEONIDI 2004 \& BABINIOTIS 2009, Dictionnaire Fondation Triandaphyllidis 1998.

7. Sur les classes des unités NN, les typologies sont nombreuses, voir entre autres, BISETTO \& SCALISE 2005, "The possible grammatical relations holding between the two constituents of a compound are basically the relations that hold in syntactic constructions: subordination, coordination and attribution.", http://www.morbocomp.sslmit.unibo.it/download/classification_of_compounds.pdf, 
consulté le 17 décembre 2015 ; SCALISE, BISETTO \& GUEVARA 2005 ; LIEBER \& ŠTEKAUER 2011 et ANASTASSIADI-SYMÉONIDI 1996 ; GAVRIILIDOU 1997 ; RALLI 2013.

8. Sur les procédés de composition nominale en grec moderne, à la base de formation des formes étudiées ici, nous renvoyons le lecteur aux travaux d'ANASTASSIADI-SYMÉONIDI 1996 ainsi qu'à l'ouvrage synthétique de RALLI 2013.

9. Sur ce phénomène en général, cf. RALLI 2013, p. 16.

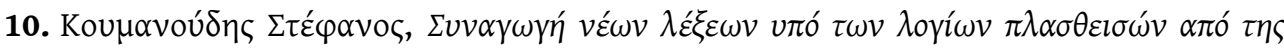

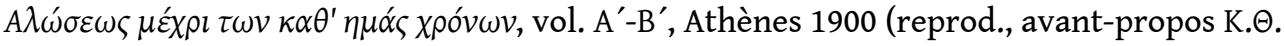
$\Delta \eta \mu \alpha \rho \alpha ́ \varsigma, 1980)$.

11. http://www.greek-language.gr/greekLang/medieval_greek/kriaras/search.html, consulté le 17 décembre 2015.

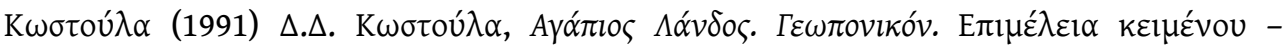

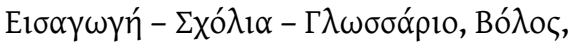
http://www.early-modern-greek.org/odigoskritikis, consulté le 17 décembre 2015.

12. Notons au passage ici l'emploi du mot simple élalo, notamment au pluriel en alternance avec $\lambda \alpha ́ \delta$. Le principe de cette alternance est étudié à travers celle entre mot

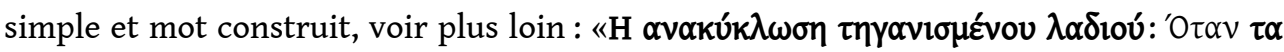

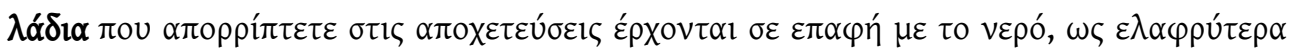

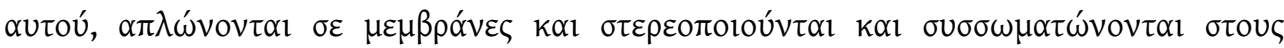

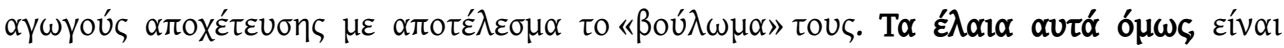

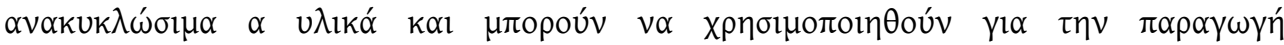

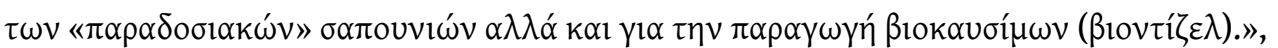
http://elialadi.blogspot.fr/2015/11/anakyklosi-tiganismenou-ladiou.html, consulté le 17 décembre 2015.

13. Cf. Ouvrage collectif de l'IREMAM, 1983, pour des données qui doivent être mises à jour.

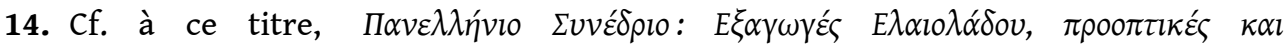

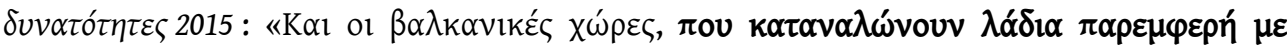

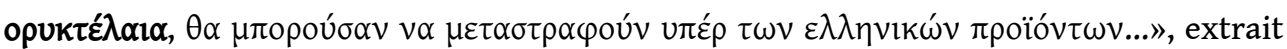
tiré du journal To $B \eta ́ \mu \alpha$,

http://www.tovima.gr/science/article/?aid=518862, consulté le 17 décembre 2015.

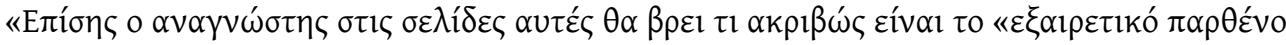

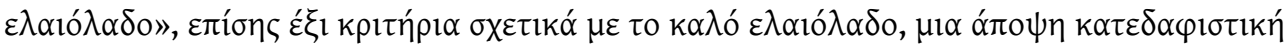

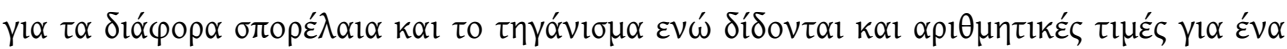

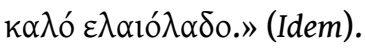

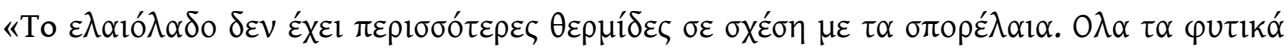

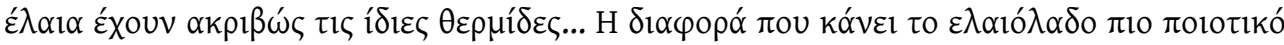

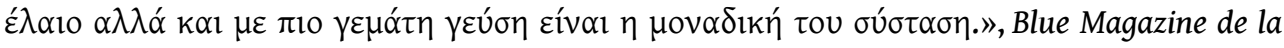
compagnie aérienne Aegean, p. 204.

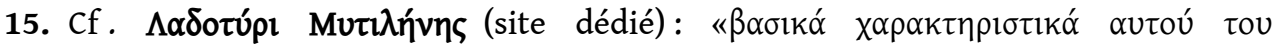

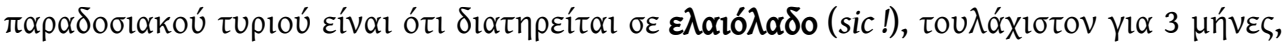

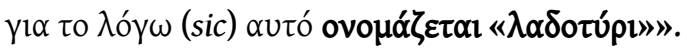

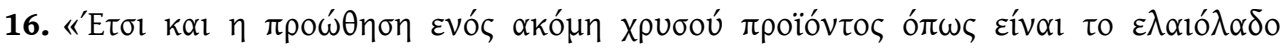

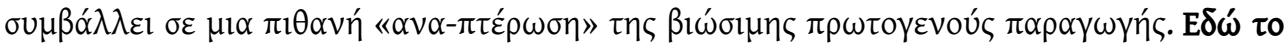

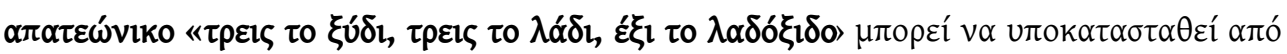




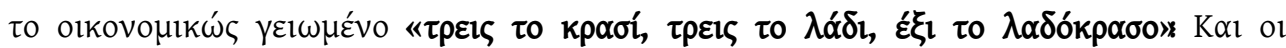

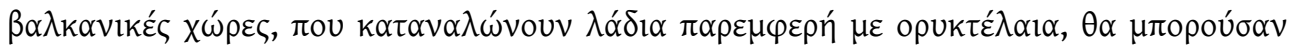

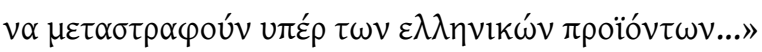

http://oikologein.blogspot.fr/2015/11/blog-post_61.html, consulté le 17 décembre 2015.

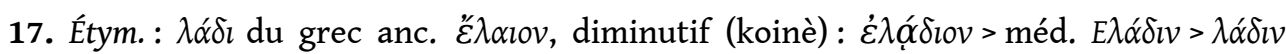
(chute de la voyelle initiale non accentuée et formation d'une syllabe $\mathrm{CV}$ ) > $\lambda \alpha \delta^{\prime}$ (chute de la consonne finale, formation d'une syllabe CV). L'huile d'olive est utilisée pour des soins corporels, à des fins médicales et rituelles ou religieuses.

Nouveau Testament : 11 occurrences de č $\lambda \alpha$ lov "used for feeding lamps", Matthieu 25, 3f, 8 : "for healing the sick", Marc 6, 13 ; Luc 10, 34 ; Jean 5, 14 : "for anointing the head and body at feasts".

18. Sur ce point, MATTHAIOU 1997.

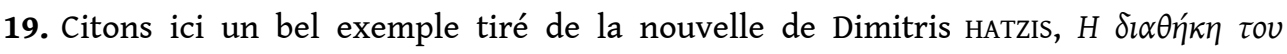

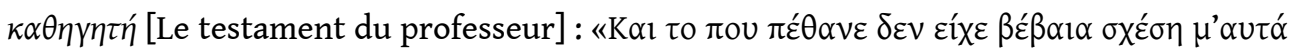

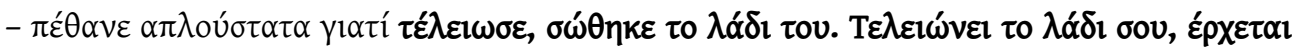

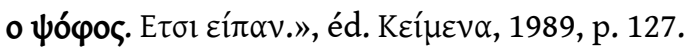

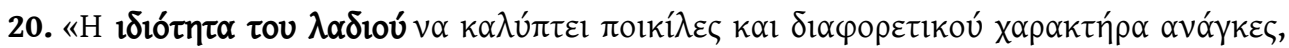

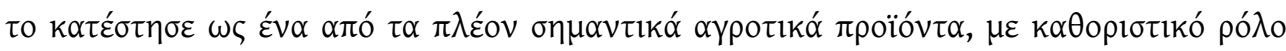

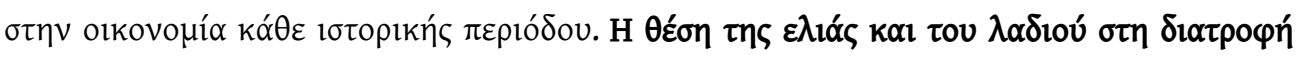

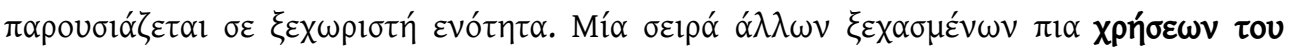

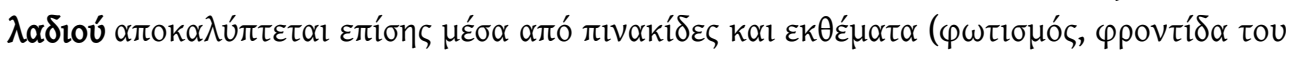

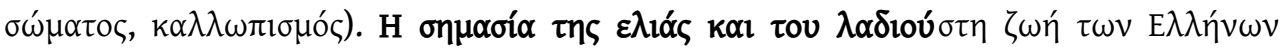

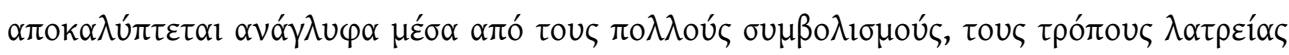

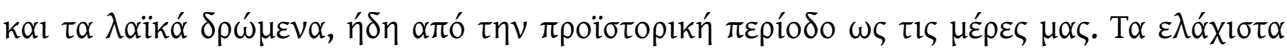

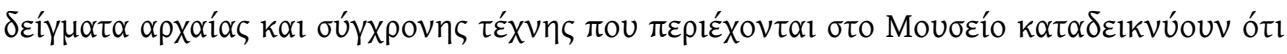

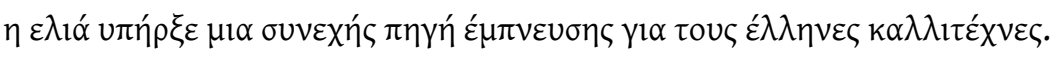

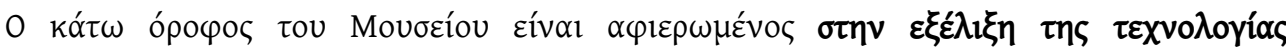

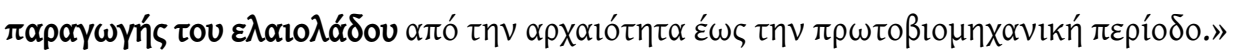

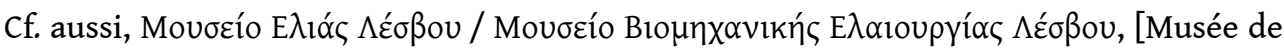

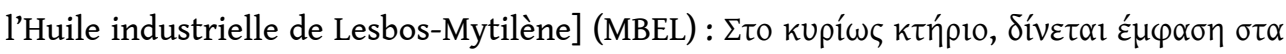

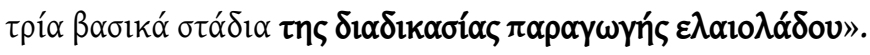

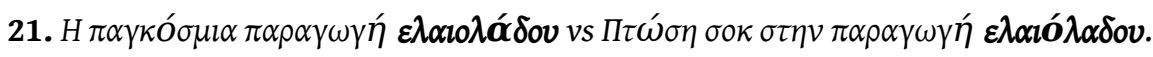

\section{RÉSUMÉS}

L'étude porte sur le mot composé $\varepsilon \lambda \alpha \imath$ io $\alpha \delta o$ « huile d'olive » en relation avec les quatre composés construits à partir des formes constitutives de ce mot, $-\varepsilon \lambda \alpha l_{0}$ - et $-\lambda \alpha \delta o-$. Dans ce cadre, est également traitée la question connexe de l'alternance dans le discours entre la forme simple $(\lambda \alpha ́ \delta l)$ "huile» et par défaut "huile d'olive» et la forme composée ainsi que la variation d'accentuation au génitif de cette dernière. Le but de cette recherche est de proposer une analyse 
du mode de construction des valeurs sémantiques et référentielles de ces unités lexicales, en relation avec les genres de discours dans lesquels elles apparaissent. L'intérêt particulier du champ sémantique lié à la nourriture, aux habitudes alimentaires à travers l'exemple de ce «pilier» de la diète méditerranéenne qu'est l'huile d'olive est d'offrir un lieu privilégié pour revenir sur la relation des catégories linguistiques avec l'extralinguistique et sur leur mode de connexion et les modalités de leur interdépendance.

The study deals with the compound word $\varepsilon \lambda \alpha$ ió $\alpha \delta \delta$ "olive oil" in relation with the four other compounds sharing the same component stems. We also consider the related question of the discourse-related alternation between the simple form $\lambda \alpha \delta_{\delta}$ "oil" (with default interpretation: "olive oil") and the compound form, as well as the variation in the stress pattern when the compound occurs in the genitive case. The semantic field of food and food habits, explored through such a key element of the Mediterranean diet as olive oil, is of special interest since it allows us to revisit the relation between linguistic categories and the extralinguistic world, and the various modalities of their connection and interdependency.

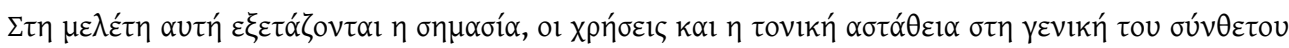

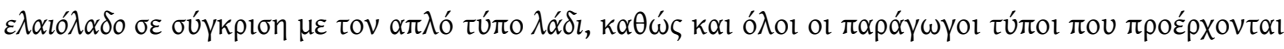

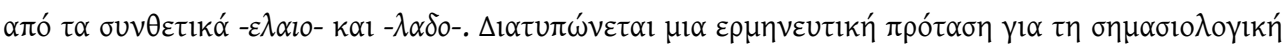

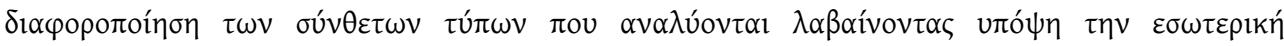

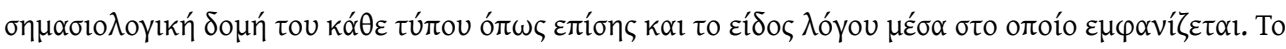

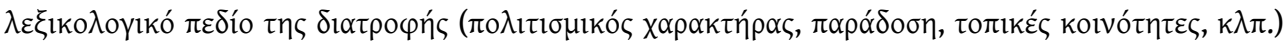

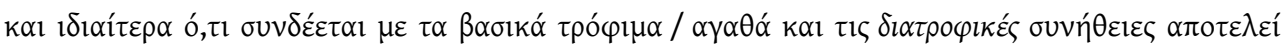

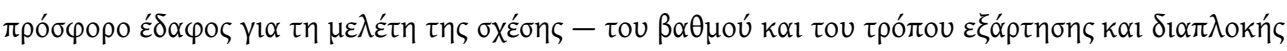

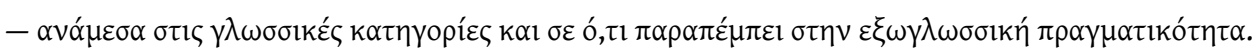

\section{INDEX}

Index géographique : Grèce

Mots-clés : huile d'olive, huile d'olive, composition nominale, composition nominale, flexion nominale, flexion nominale, variation lexicale, variation lexicale, registre de langue, registre de langue

Keywords : Olive oil, Nominal composition, Nominal bending, Lexical variation, Language Register, Greece, Twenty First century, Linguistics

motsclesmk МАСЛИНОВО МАСЛО, ГРЦИЈА, ДВАЕСЕТ И ПРВИОТ ВЕК, ЛИНГВИСТИКА

Thèmes : Linguistique

motsclestr Zeytinyağı, Yunanistan, Yirmi birinci yüzyıl, Dilbilim

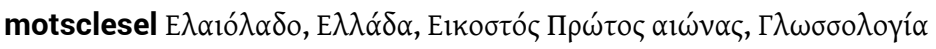

Index chronologique : vingt-et-unième siècle

\section{AUTEUR}

\section{SOPHIE VASSILAKI}

Inalco, SeDyL, USPC, CNRS, IRD 